\title{
A 2.3GHz LC-tank CMOS VCO with optimal phase noise performance
}

\author{
Andreani, Pietro; Fard, Ali
}

Published in:

2006 IEEE International Solid-State Circuits Conference, Digest of Technical Papers

Link to article, DOI:

10.1109/ISSCC.2006.1696108

Publication date:

2006

Document Version

Publisher's PDF, also known as Version of record

Link back to DTU Orbit

Citation (APA):

Andreani, P., \& Fard, A. (2006). A 2.3GHz LC-tank CMOS VCO with optimal phase noise performance. In 2006 IEEE International Solid-State Circuits Conference, Digest of Technical Papers (pp. 691-700). IEEE.

https://doi.org/10.1109/ISSCC.2006.1696108

\section{General rights}

Copyright and moral rights for the publications made accessible in the public portal are retained by the authors and/or other copyright owners and it is a condition of accessing publications that users recognise and abide by the legal requirements associated with these rights.

- Users may download and print one copy of any publication from the public portal for the purpose of private study or research.

- You may not further distribute the material or use it for any profit-making activity or commercial gain

- You may freely distribute the URL identifying the publication in the public portal

If you believe that this document breaches copyright please contact us providing details, and we will remove access to the work immediately and investigate your claim. 


\subsection{A 2.3GHz LC-Tank CMOS vCO with Optimal Phase Noise Performance}

Pietro Andreani ${ }^{1}$, Ali Fard ${ }^{2}$

${ }^{1}$ Technical University of Denmark, Kgs. Lyngby, Denmark ${ }^{2}$ Mälardalen University, Västerås, Sweden

It might appear surprising that something new can still be found about fundamental phase noise relations in LC-tank CMOS VCOs. Yet, this work demonstrates that the $1 / f^{2}$ phase noise displayed by the current-biased VCO with double switch pair (Fig. 11.2.1, referred to hereafter as the DS-VCO) is lower than is commonly assumed. In particular, its minimum level (i.e., that caused by tank losses and switches) is $6 \mathrm{~dB}$ below the minimum level for the LC-tank VCO with a single switch pair and centertapped inductor (SS-VCO), when both VCOs make use of the same LC tank and bias current $I_{B}$, and both are working in the current-limited region. Such a result was already conjectured in [1], noticing that, under the above conditions, the oscillation amplitude in the DS-VCO is ideally double that in the SS-VCO. This conjecture, however, hinged on the unproven (and actually unmotivated) assumption that the two VCO cores generate an equal amount of noise (see e.g. [1], reply section). The best previous understanding of the DS-VCO was that the thermal noise in the transistors' channels dominates the $1 / f^{2}$ phase noise [2]. Since such noise sources ideally contribute only $\sim 40 \%$ of the total phase noise in the SS-VCO [3], it would seem that the DS-VCO cannot yield a full $6 \mathrm{~dB}$ phase noise improvement and would, therefore, have a lower phase-noise figure-of-merit (FoM) than the SS-VCO. This conclusion may be the main reason why the DS-VCO is less popular than the SS-VCO, in spite of the lower $I_{B}$ needed for a given oscillation amplitude and the well-known opportunity to achieve a low $1 / f^{3}$ corner with a DS-VCO.

Our phase noise analysis makes use of a symbolic approach to Hajimiri's and Lee's impulse sensitivity function (ISF) theory [4], where the $1 / f^{2}$ phase noise $L$ at the angular frequency offset $\Delta \omega$ is calculated as

$$
L(\Delta \omega)=10 \log \left(\sum_{n} \frac{\Gamma_{n, \mathrm{rms}}^{2} \cdot \overline{i_{n}^{2}} / \Delta f}{2 q_{\max }^{2}(\Delta \omega)^{2}}\right)
$$

where $q_{\max }$ is the maximum amount of signal charge loaded onto the tank capacitance $C, i_{n}$ is a white current noise source, and $\Gamma_{n}$ is the (effective) ISF for $i_{n}$. It is well-known [3,4] that, assuming a sinusoidal waveform $V(\phi)=A \sin (\phi)$ across $C$ (with $0 \leq \phi \leq 2 \pi$ ), the ISF associated with $R$ is $\Gamma_{R}(\phi)=\cos (\phi)$. The symbolic expressions for the ISF for all MOS channel-current noise sources can be found in terms of the known $\Gamma_{R}(\phi)$ by analyzing the simplified small-signal circuit of the oscillator (Fig. 11.2.2) and applying the definition of ISF as the excess phase generated by a current impulse injected into the oscillator in parallel with the respective noise source [4]. A straightforward circuit analysis, hinging on the property that a capacitor presents infinitesimal impedance to an impulsive current, results in the ISFs given in Fig. 11.2.2. A compact formula for the $1 / f^{2}$ phase noise is obtained using the square-law MOS current equation, and a MOS current noise $\overline{i_{\mathrm{n}, \mathrm{n} / \mathrm{p}}^{2}}=4 k_{B} T \gamma_{n / p} g_{m, n / p} \Delta f$, where $\gamma_{n}\left(\gamma_{p}\right)$ is the channel noise factor for nMOS (pMOS) devices. Under these hypotheses, the exact expression for the $1 / f^{2}$ phase noise caused by tank losses and switches is

$$
L(\Delta \omega)=10 \log \left(\frac{k_{B} T}{A^{2} C^{2}(\Delta \omega)^{2} R}\left(1+\frac{\gamma_{n}+\gamma_{p}}{2}\right)\right)
$$

where $A \approx(4 / \pi) R I_{B}$ is the oscillation amplitude. The impact of the tail current noise can be derived in the same way as in [3].

A careful comparison with the $1 / \mathrm{f}^{2}$ phase noise displayed by the SS-VCO [3] shows indeed that (2) yields a $\sim 6 \mathrm{~dB}$ lower phase noise for the same LC-tank and $I_{B}\left(6 \mathrm{~dB}\right.$ exactly if $\left.\gamma_{p}=\gamma_{n}\right)$. We notice that the noise contribution of each transistor in the DS-VCO is proportional to $1 / \mathrm{R}$ independently of the transistor transconductance, which is true in the SS-VCO as well. However, the relative impact of each transistor in the DS-VCO is only half that in the SS-VCO, and, since there are twice as many transistors in the DS-VCO, the total relative impact of the transistors is the same in both oscillators. Numerous SpectreRF simulations fully confirm these results.

Although (2) shows that nMOS and pMOS switches ideally contribute equally to phase noise, there is nevertheless an asymmetry between nMOS and pMOS pairs, due to the absence of a high impedance level at the sources of the pMOS switches (nMOS and pMOS swap of course roles if top-biasing is adopted). As a consequence, while the nMOS ISFs are independent of the capacitances $\mathrm{C}_{\mathrm{par}}$ between LC-tank outputs and ground, the pMOS ISFs depend very strongly on $\mathrm{C}_{\mathrm{par}}$. A large $\mathrm{C}_{\mathrm{par}}$ results in much larger pMOS ISFs (Fig. 11.2.3), and the pMOS noise rapidly becomes the dominant cause of phase noise. As an example, if half of the overall tank capacitance is parasitic, the simulated phase noise deterioration, compared to (2), is already in excess of $3 \mathrm{~dB}$.

It is therefore critical to keep $\mathrm{C}_{\mathrm{par}}$ as small as possible, by minimizing transistor dimensions (especially pMOS), capacitances of coil and varactors to substrate, and loads presented by VCO buffers.

The DS-VCO was built in a $0.35 \mu \mathrm{m}$ CMOS process with four $\mathrm{Al}$ metal layers. The top layer is $2 \mu \mathrm{m}$ thick, allowing the realization of a $2.6 \mathrm{nH}$ inductor with a $\mathrm{Q}$ of $\sim 11$ at $2.3 \mathrm{GHz}$. No shield under the inductor was used, in order to minimize $\mathrm{C}_{\mathrm{par}}$. Floating MIM capacitors and accumulation-MOS varactors realize the mixedmode tuning, setting at the same time the center frequency to $2.25 \mathrm{GHz}$. Open-drain buffers drive $50 \Omega$ off-chip loads. A reference SS-VCO was implemented as well, making use of an nMOS pair and of the same tank used in the DS-VCO. The phase noise induced by the tail current noise was made negligible in both VCOs.

The DS-VCO is tunable between $2.15 \mathrm{GHz}$ and $2.35 \mathrm{GHz}$, covered in four overlapping bands of $75 \mathrm{MHz}$ each. Fig. 11.2.4 shows the phase noise measured as a function of $\mathrm{I}_{\mathrm{B}}$; the phase noise difference in favor of the DS-VCO is $5-7 \mathrm{~dB}$ when both VCOs are working in the current-limited region, in very good agreement with the theoretical $6 \mathrm{~dB}$. The phase noise plot for the DS-VCO at 2.3GHz (Fig. 11.2.5) shows a phase noise of $-143.9 \mathrm{dBc} / \mathrm{Hz}$ at $3 \mathrm{MHz}$ offset, for an excellent $\mathrm{FoM}$ of $191.6 \mathrm{dBc} / \mathrm{Hz}\left(\mathrm{V}_{\mathrm{dd}}=2.5 \mathrm{~V}\right.$, $\mathrm{I}_{\mathrm{B}}=4 \mathrm{~mA}$ ). Despite the process used, this is the highest FoM reported to date for DS-VCOs. The $1 / \mathrm{f}^{3}$ corner is below $250 \mathrm{kHz}$. Fig. 11.2.6 shows that the DS-VCO phase noise varies by less than $1 \mathrm{~dB}$ across the tuning range, the FoM being almost constant. Finally, the phase noise is at most only $1 \mathrm{~dB}$ higher than the value yielded by (2), from which it can be concluded that the phase noise performance of the DS-VCO is indeed optimal. A chip photo of both VCOs is visible in Fig. 11.2.7.

References:

[1] H. Wang, A. Hajimiri, T. H. Lee. "Comments to "Design Issues in CMOS Differential LC Oscillators," and Authors' Reply," IEEE J. Solid-State Circuits, vol. 35, no. 2, pp. 286-287, Feb., 2000.

[2] D. Ham , A. Hajimiri. "Concepts and Methods in Optimization of Integrated LC VCOs," IEEE J. Solid-State Circuits, vol. 36, no. 6, pp. 896909, June, 2001.

[3] P. Andreani et al. "A Study of Phase Noise in Colpitts and LC-Tank CMOS Oscillators," IEEE J. Solid-State Circuits, vol. 40, no. 5, pp. 11071118, May, 2005.

[4] A. Hajimiri, T. H. Lee. "A General Theory of Phase Noise in Electrical Oscillators," IEEE J. Solid-State Circuits, vol. 33, no. 2, pp. 179-194, Feb., 1998. 


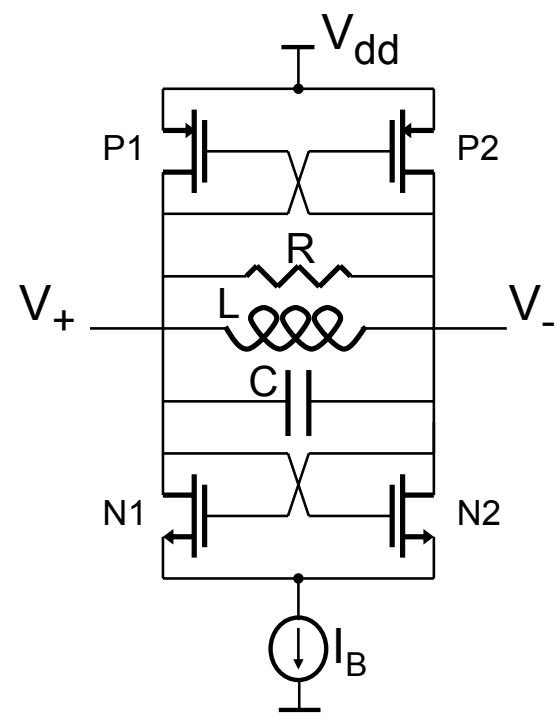

Figure 11.2.1: Simplified schematic view of the DS-VCO.

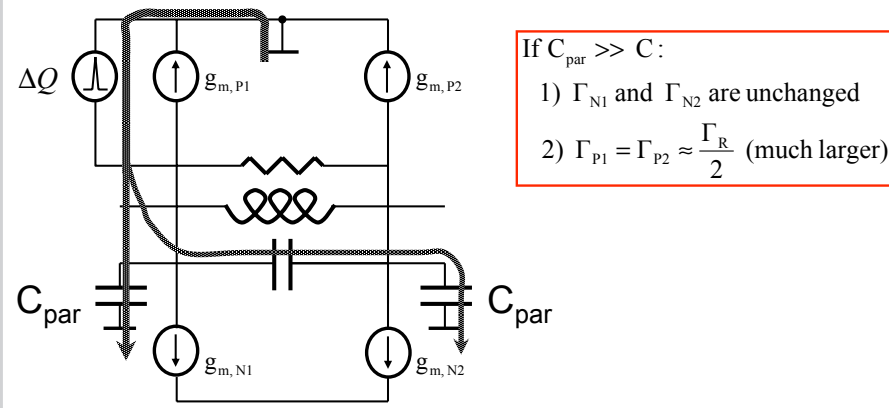

Figure 11.2.3: The symmetry between nMOS and pMOS pairs is broken when $\mathrm{C}_{\mathrm{par}}$ is non-negligible. If $\mathrm{C}_{\mathrm{par}}$ is large, pMOS-induced phase noise becomes dominant.

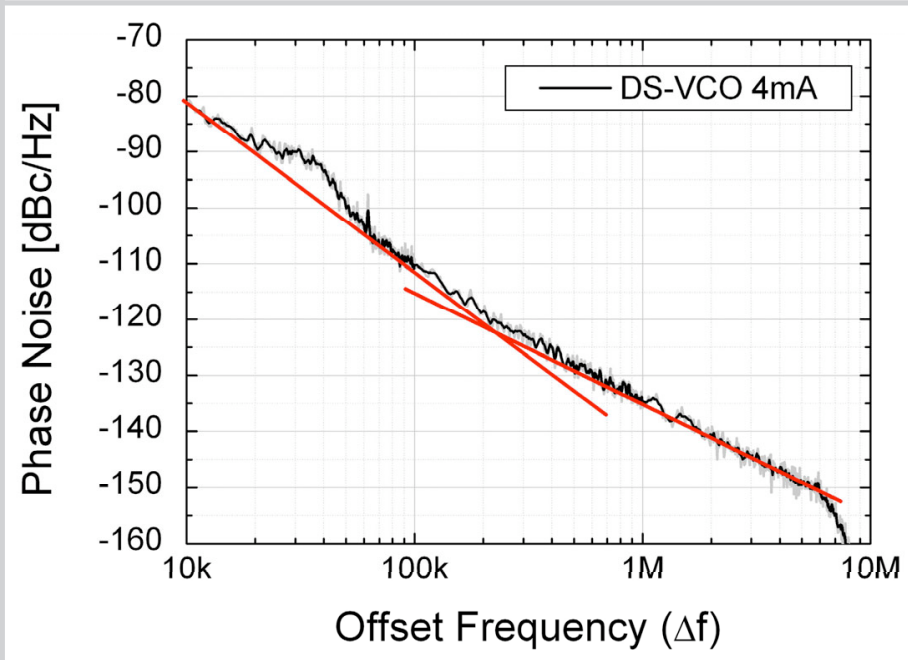

Figure 11.2.5: Phase noise plot for the DS-VCO $\left(f_{o s c}=2.3 G H z, V_{d d}=2.5 V, I_{B}=4 m A\right)$.

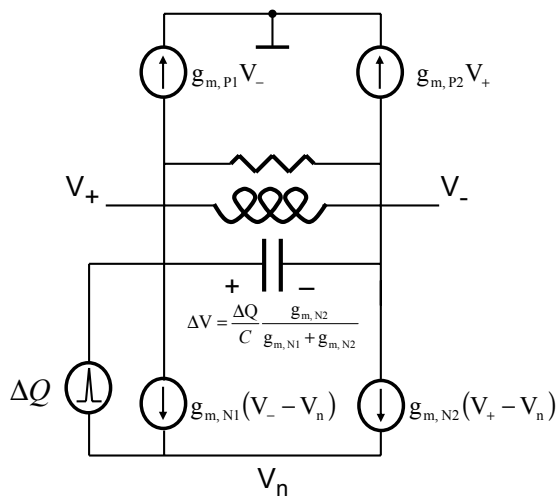

$\Gamma_{\mathrm{R}}=\cos (\phi)$
$\Gamma_{\mathrm{N} 1}=\Gamma_{\mathrm{R}} \frac{\mathrm{g}_{\mathrm{m}, \mathrm{N} 2}}{\mathrm{~g}_{\mathrm{m}, \mathrm{N} 1}+\mathrm{g}_{\mathrm{m}, \mathrm{N} 2}}$
$\Gamma_{\mathrm{N} 2}=\Gamma_{\mathrm{R}} \frac{\mathrm{g}_{\mathrm{m}, \mathrm{N} 1}}{\mathrm{~g}_{\mathrm{m}, \mathrm{N} 1}+\mathrm{g}_{\mathrm{m}, \mathrm{N} 2}}$
$\Gamma_{\mathrm{P} 1}=\Gamma_{\mathrm{R}} \frac{\mathrm{g}_{\mathrm{m}, \mathrm{P} 2}}{\mathrm{~g}_{\mathrm{m}, \mathrm{P} 1}+\mathrm{g}_{\mathrm{m}, \mathrm{P} 2}}$
$\Gamma_{\mathrm{P} 2}=\Gamma_{\mathrm{R}} \frac{\mathrm{g}_{\mathrm{m}, \mathrm{P} 1}}{\mathrm{~g}_{\mathrm{m}, \mathrm{P} 1}+\mathrm{g}_{\mathrm{m}, \mathrm{P} 2}}$

Figure 11.2.2: Left: small-signal circuit for phase noise calculations (as an example, the impulsive current source in parallel to $\mathrm{N} 1$ is used to derive the ISF for N1). Right: expressions of all relevant ISFs (all transconductances are functions of $\phi$ ).

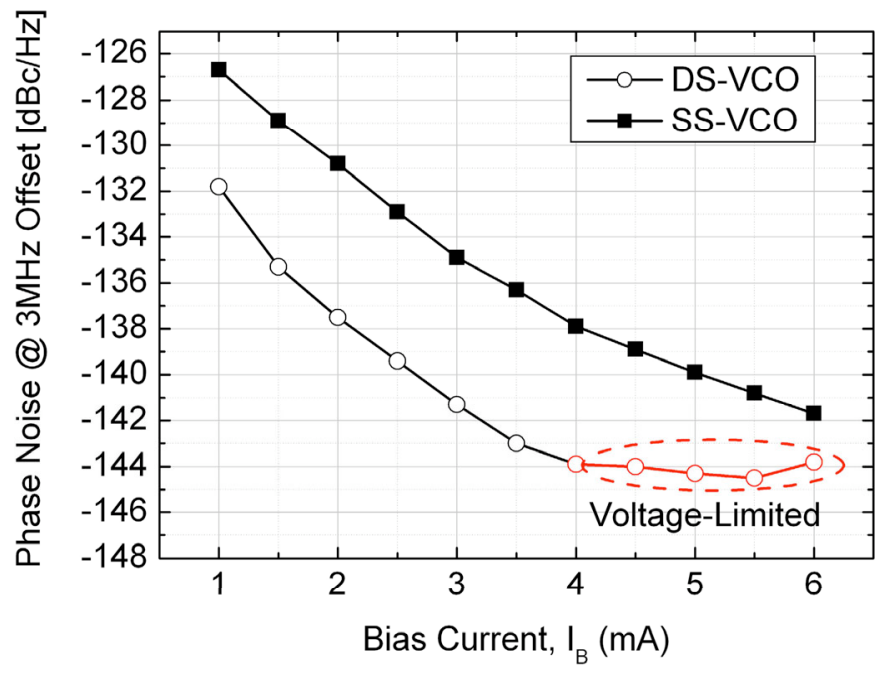

Figure 11.2.4: Current-limited phase noise of SS-VCO and DS-VCO at $3 \mathrm{MHz}$ frequency offset, as a function of bias current.

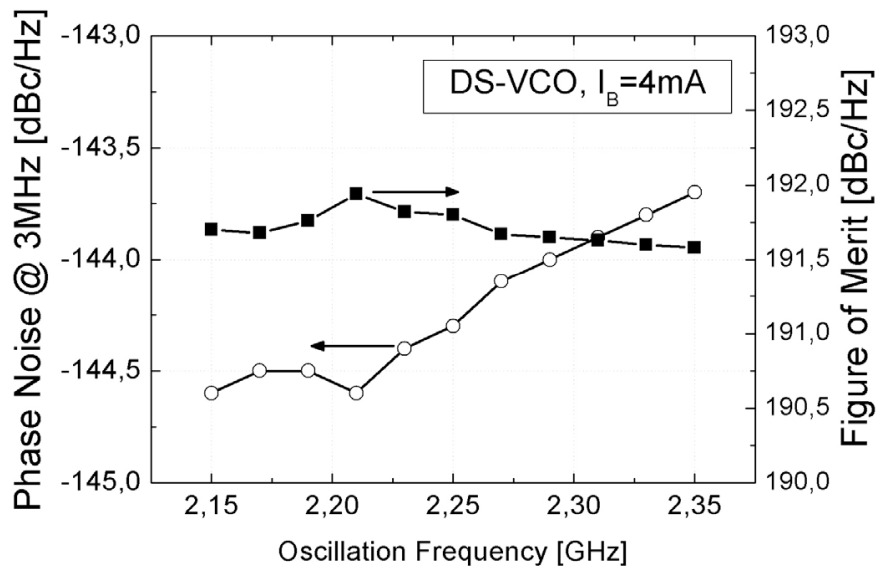

Figure 11.2.6: Phase noise and FoM for the DS-VCO at $3 \mathrm{MHz}$ frequency offset across the tuning range $\left(\mathrm{V}_{\mathrm{dd}}=2.5 \mathrm{~V}, \mathrm{I}_{\mathrm{B}}=4 \mathrm{~mA}\right)$. 
ISSCC 2006 / SESSION 11 / RF BUILDING BLOCKS AND PLLS / 11.2

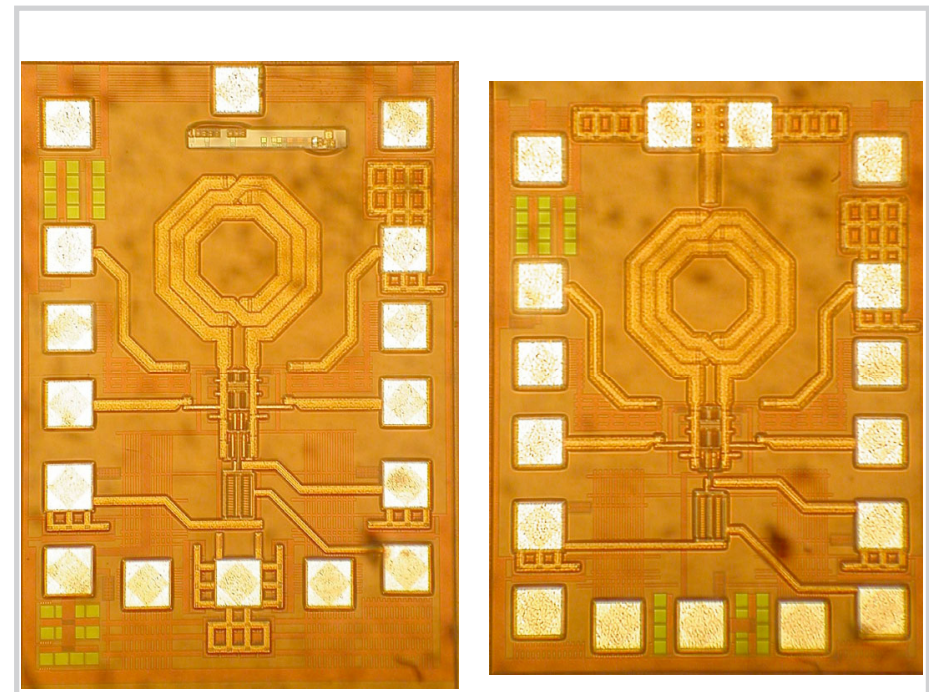

Figure 11.2.7: Chip photograph of DS-VCO (left) and SS-VCO (right).

- 2006 IEEE International Solid-State Circuits Conference 


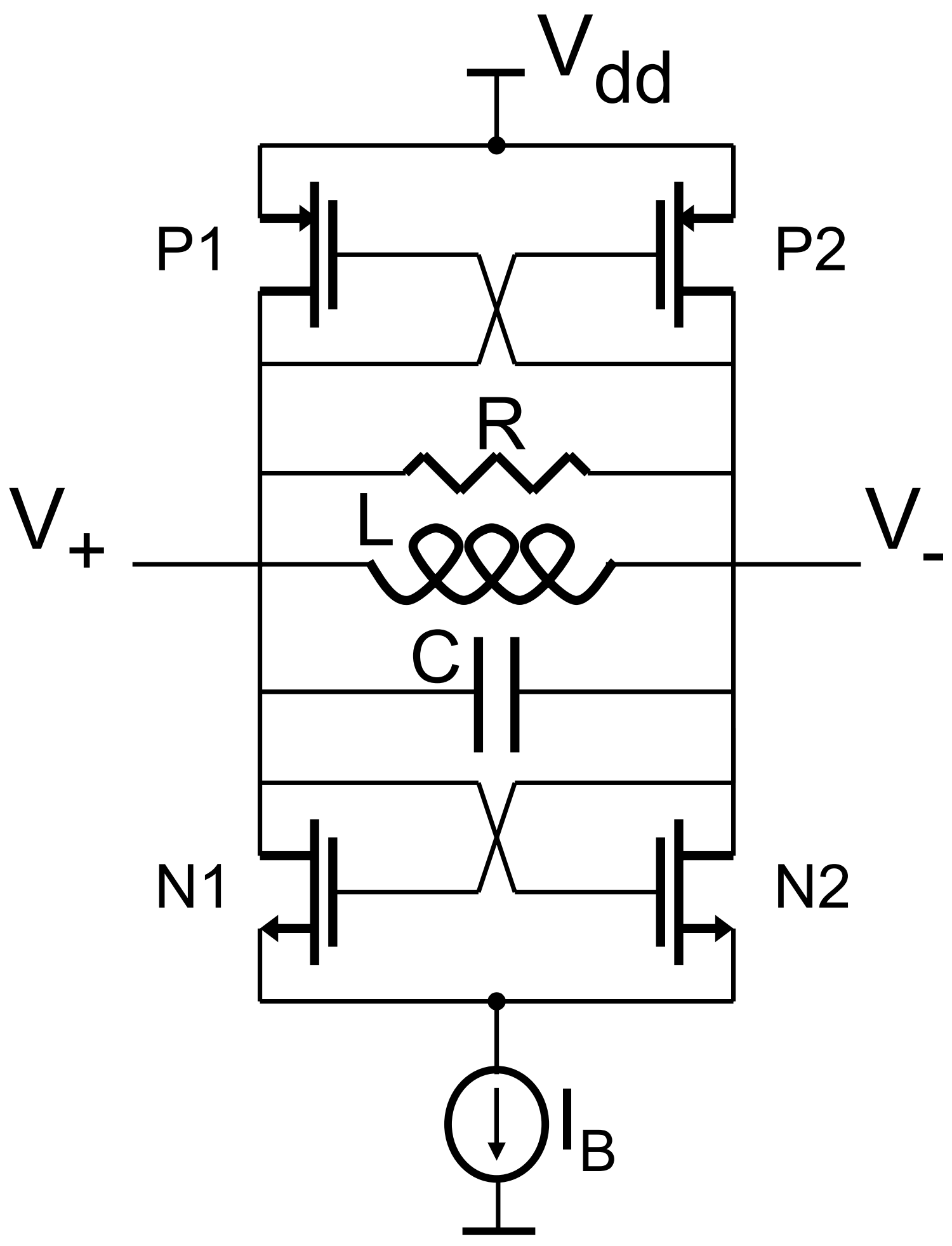

Figure 11.2.1: Simplified schematic view of the DS-VCO. 


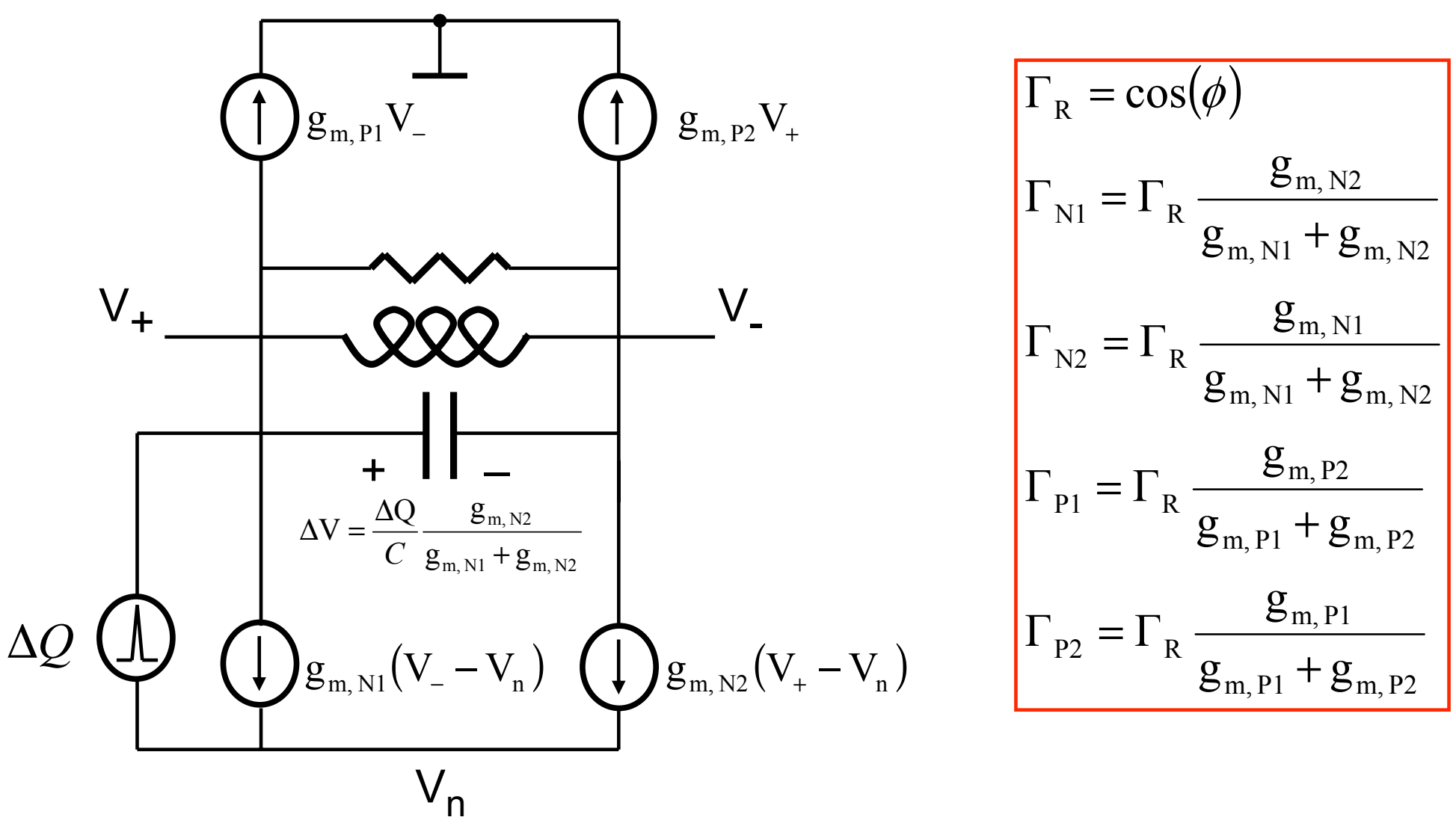

Figure 11.2.2: Left: small-signal circuit for phase noise calculations (as an example, the impulsive current source in parallel to $\mathbf{N} 1$ is used to derive the ISF for N1). Right: expressions of all relevant ISFs (all transconductances are functions of $\phi$ ). 


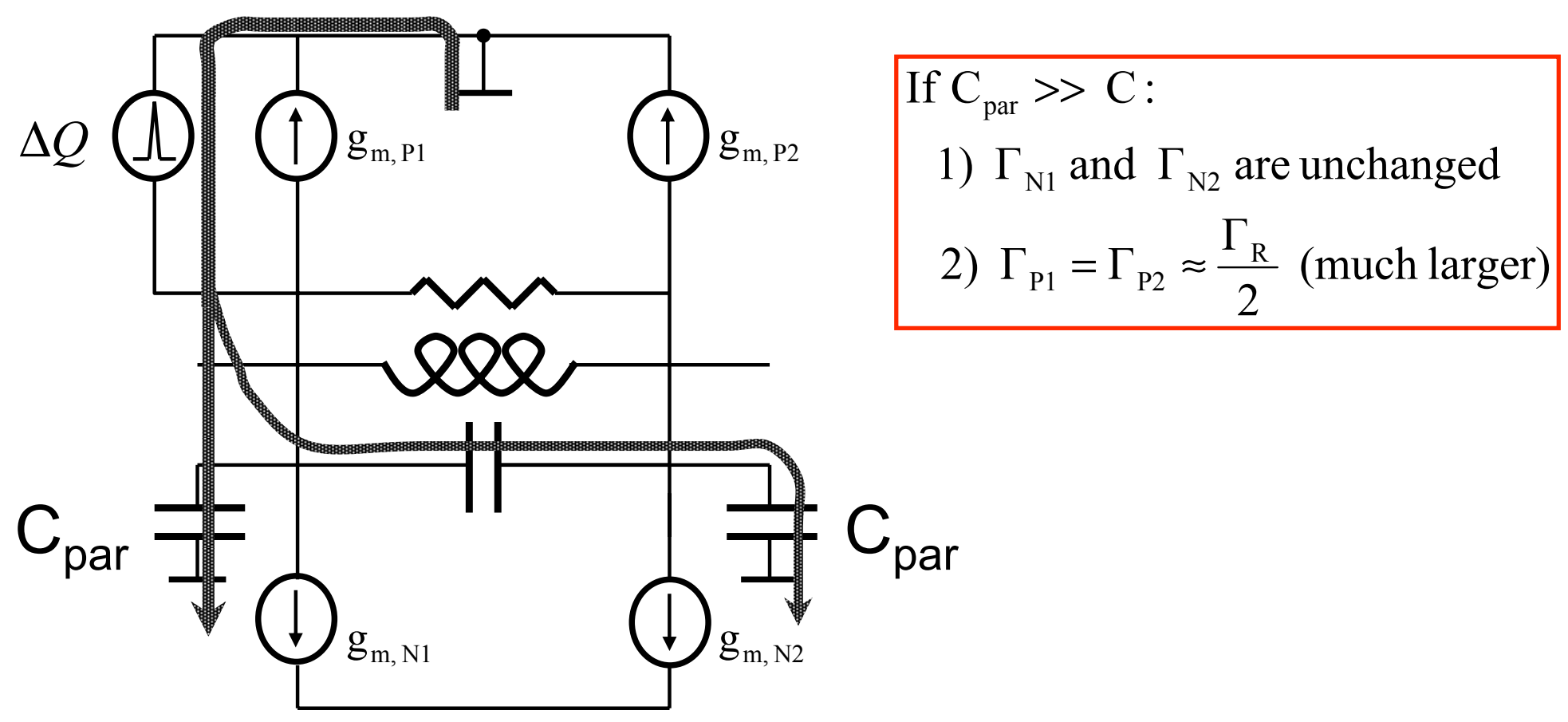

Figure 11.2.3: The symmetry between nMOS and pMOS pairs is broken when $C_{p a r}$ is non-negligible. If $C_{p a r}$ is large, pMOS-induced phase noise becomes dominant. 


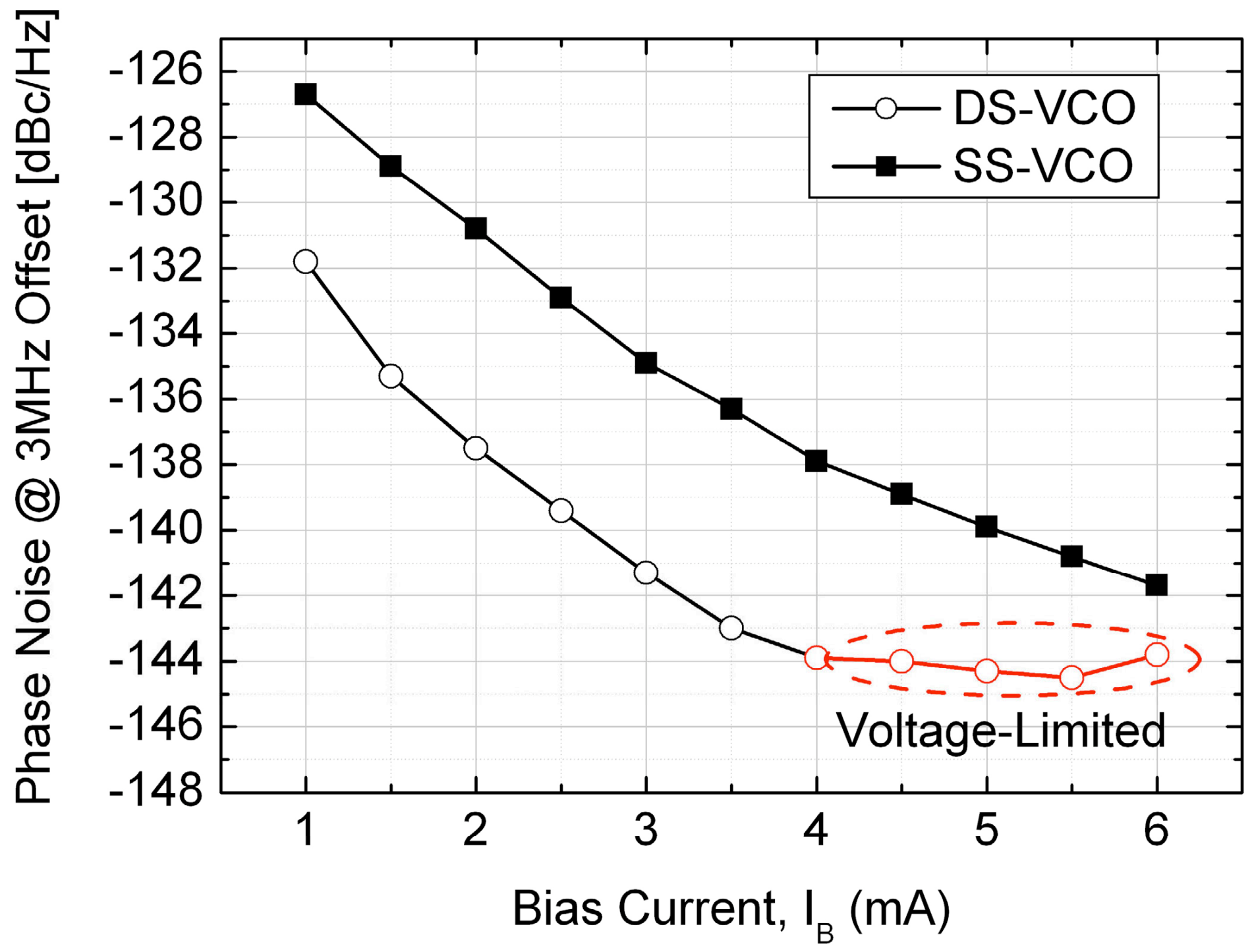

Figure 11.2.4: Current-limited phase noise of SS-VCO and DS-VCO at 3MHz frequency offset, as a function of bias current. 


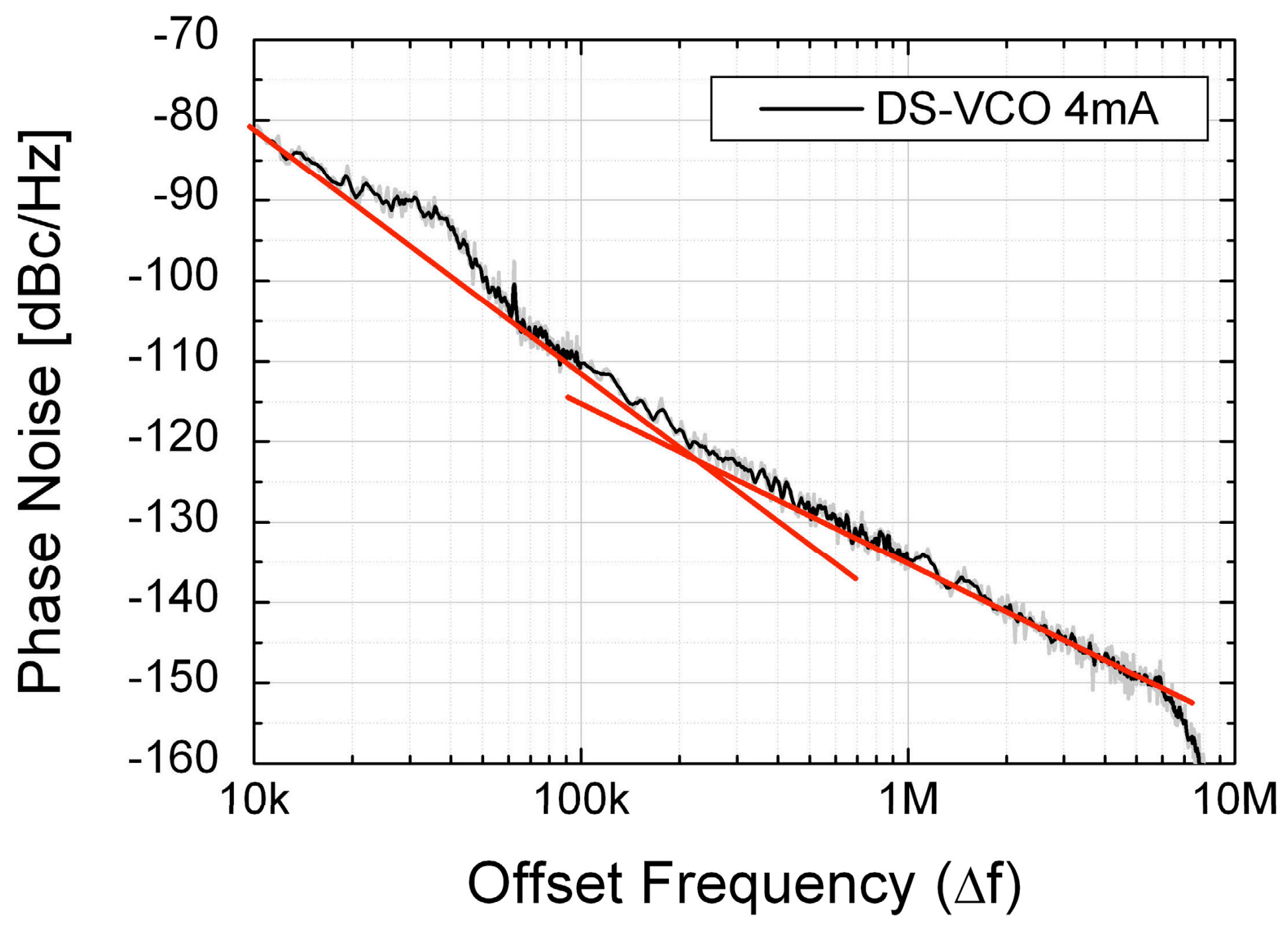

Figure 11.2.5: Phase noise plot for the DS-VCO $\left(f_{o s c}=2.3 G H z, V_{d d}=2.5 V, I_{B}=4 m A\right)$. 


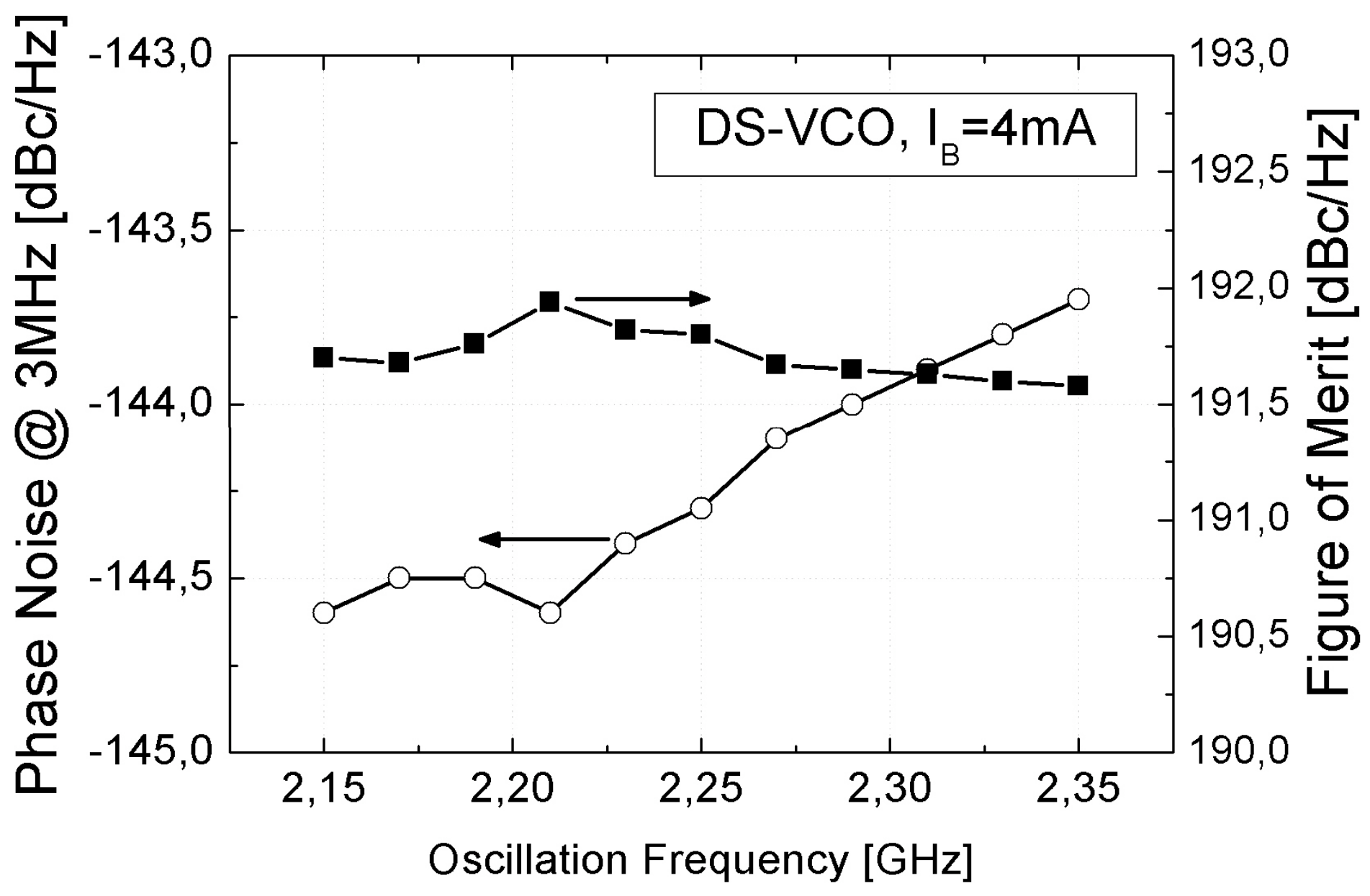

Figure 11.2.6: Phase noise and FoM for the DS-VCO at 3MHz frequency offset across the tuning range $\left(V_{d d}=2.5 V, I_{B}=4 m A\right)$. 

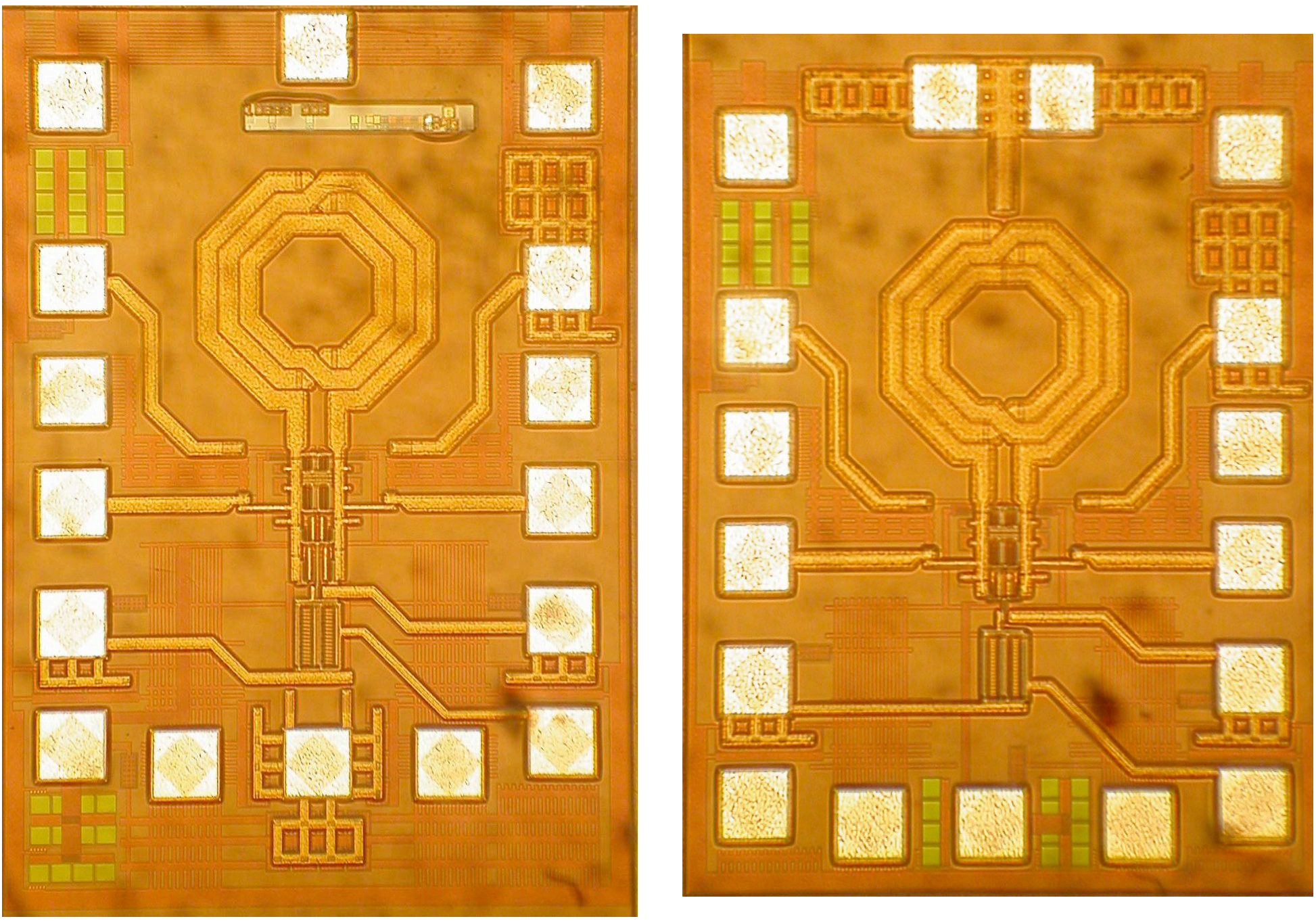

Figure 11.2.7: Chip photograph of DS-VCO (left) and SS-VCO (right).

- 2006 IEEE International Solid-State Circuits Conference 